\title{
On a CR Transversality Problem Through the Approach of the Chern-Moser Theory
}

\author{
Xiaojun Huang • Yuan Zhang
}

Received: 3 September 2011 / Published online: 7 April 2012

(C) Mathematica Josephina, Inc. 2012

\begin{abstract}
In this paper, we give a geometric condition for a CR map, which sends a $\mathrm{CR}$ non-umbilical Levi non-degenerate hypersurface in $\mathbb{C}^{n+1}$ into the hyperquadric in $\mathbb{C}^{n+2}$ with the same signature, to be CR transversal.
\end{abstract}

Keywords CR transversality $\cdot$ Chern-Moser theory $\cdot$ CR umbilical $\cdot$ Levi non-degeneracy $\cdot$ Signature $\cdot$ Levi-cone

Mathematics Subject Classification $32 \mathrm{~V} 30 \cdot 32 \mathrm{H} 02$

\section{Introduction}

The study of CR transversality (or the Hopf lemma property) has found applications in understanding the regularity and rigidity phenomena for CR mappings in the recent development of several complex variables. Generally speaking, the CR transversality problem asks if a CR map sending a piece of hypersurface into another one is either totally degenerate (namely, mapping an open subset of the source space into the target hypersurface) or has a non-vanishing normal derivative (which in many situations is equivalent to the local immersion property of the map). When the hypersurfaces are pseudoconvex, the classical Hopf lemma is applicable. However, the situation in the non-pseudoconvex case is much more subtle. When the hypersurfaces are sitting in the same complex space, there has been much work done along these lines.

Communicated by Alexander Isaev.

X. Huang

Department of Mathematics, Rutgers University, New Brunswick, NJ 08903, USA

e-mail: huangx@math.rutgers.edu

Y. Zhang ( $ه)$

Department of Mathematical Sciences, Indiana University - Purdue University, Fort Wayne, IN 46805-1499, USA

e-mail: yuz009@math.ucsd.edu 
Here, we only refer the reader to the work of Pinchuk [18], Fornaess [12], BaouendiRothschild [5], Ebenfelt-Rothschild [10] for smooth CR mappings, and Huang [14] for multiple-valued holomorphic maps (holomorphic correspondences), as well as many references quoted in $[5,10,14]$.

The study in the non-pseudoconvex case with higher codimensions started with the work of Baouendi-Huang [3], where the CR transversality is obtained for CR mappings sending a piece of a hyperquadric into another hyperquadric with the same signature. In the work of Baouendi-Ebenfelt-Rothschild [2], it is proved in a very general setting that the transversality holds at least along the complements of proper real analytic subsets, which may be different for different maps under consideration. (See also a recent preprint [11], which further generalizes the work of [2] in some settings.) However, except in the case dealt with in Baouendi-Huang [3], it is an open question to understand under what circumstances the CR transversality in the higher co-dimensions holds everywhere along the source manifold. For instance, the following easily stated conjecture is open.

Conjecture Let $M_{1} \subset \mathbb{C}^{n+1}$ and $M_{2} \subset \mathbb{C}^{N+1}$ be two (connected) Levi nondegenerate real analytic hypersurfaces with the same signature $\ell>0$. Let $F$ be a smooth CR map sending $M_{1}$ into $M_{2}$. Then either $F$ is a local immersion along $M_{1}$ or $F$ sends an open neighborhood $U$ of $M_{1}$ in $\mathbb{C}^{n+1}$ into $M_{2}$.

In an earlier paper of the authors [15], we demonstrated that many mapping properties are related to the behavior of the Chern-Moser-Weyl tensors of the hypersurfaces along the Levi cone. In this paper, we show that these CR invariants, together with the work of Meylan-Mir-Zaitsev [17] on the convergence of (non-degenerate) formal maps into hyperquadrics, can also be used to work on the CR transversality problem. Our method is quite different from what is used in the previous related work.

Next, we set up some notation to state precisely our main result. Given two CR hypersurfaces $M \subset \mathbb{C}^{n+1}, \tilde{M} \subset \mathbb{C}^{N+1}$ and a smooth $\mathrm{CR}$ map $F: M \rightarrow \tilde{M}, F$ is said to be $C R$ transversal at $p \in M$ if

$$
T_{F(p)}^{(1,0)} \tilde{M}+F_{*}\left(T_{p}^{(1,0)} \mathbb{C}^{n+1}\right)=T_{F(p)}^{(1,0)} \mathbb{C}^{N+1},
$$

where $T_{p}^{(1,0)} \mathbb{C}^{n+1}$ and $T_{F(p)}^{(1,0)} \tilde{M}$ denote the tangent spaces of type $(1,0)$ for $\mathbb{C}^{n+1}$ and $\tilde{M}$ at $p$ and $F(p)$, respectively. Note that according to the above definition, a CR map being CR transversal at $p \in M$ is equivalent to the nonvanishing of the derivative of its normal component at $p$ along the normal direction (see [2], for example). Notice also that when both $M$ and $\tilde{M}$ are strongly pseudoconvex, any non-constant smooth CR map between them is always CR transversal, by an application of the classical Hopf lemma.

Now, let $M_{\ell}$ be a Levi non-degenerate smooth hypersurface of signature $\ell>0$. Let $p \in M$. After a holomorphic change of coordinates, we may assume that $p=0$ and $M_{\ell}$ near $p=0$ is defined by an equation of the form

$$
M_{\ell}:=\left\{(z, w) \in \mathbb{C}^{n} \times \mathbb{C}: \rho=-\Im w-\sum_{j=1}^{\ell}\left|z_{j}\right|^{2}+\sum_{j=\ell+1}^{n}\left|z_{j}\right|^{2}+o(3)=0\right\} .
$$


When the terms with degree three or higher in the defining equation for $M_{\ell}$ can be made to be zero, we get the hyperquadric $H_{\ell}^{n+1}$ with signature $\ell$. Namely, we have

$$
H_{\ell}^{n+1}:=\left\{(z, w) \in \mathbb{C}^{n} \times \mathbb{C}: \rho=-\Im w-\sum_{j=1}^{\ell}\left|z_{j}\right|^{2}+\sum_{j=\ell+1}^{n}\left|z_{j}\right|^{2}=0\right\} .
$$

We always assume that $\ell \leq n / 2$ to make $\ell$ an invariant. We say $0 \in M_{\ell}$ is a $C R$ umbilical point if the Chern-Moser-Weyl curvature tensor vanishes at $p=0$ (see [6] or [15]). Namely, if 0 is CR umbilical, then there is a holomorphic change of coordinates such that in the new coordinates, we can make $\rho=-\Im w-\sum_{j=1}^{\ell}\left|z_{j}\right|^{2}+$ $\sum_{j=\ell+1}^{n}\left|z_{j}\right|^{2}+o(4)$.

We now state the main theorem of this paper.

Theorem 1.1 Let $M_{\ell}$ be a smooth Levi non-degenerate hypersurface of signature $\ell$ in $\mathbb{C}^{n+1}, n \geq 2$. Assume that $0 \in M_{\ell}$ is not $C R$ umbilical. If $F$ is a holomorphic map defined in a small neighborhood $U$ of $0 \in \mathbb{C}^{n+1}$ such that $F\left(M_{\ell} \cap U\right) \subset H_{\ell}^{(n+1)+1}$, then either $F$ is $C R$ transversal to $M_{\ell}$ at 0 , or $F(U) \subset H_{\ell}^{(n+1)+1}$.

It might be interesting to notice that usually, to apply the classical Hopf lemma, the sign condition is imposed on the degree-two terms in the defining functions of the target manifolds, while the sign condition here is imposed on the fourth-order degree terms (along the Levi-cone direction) for the defining functions of the source manifolds.

The rest of the paper is organized as follows: In Sects. 2 and 3, we set up more notation and give some background material. We then prove some preliminary lemmas. In Sect. 4, we give the proof of the main Theorem 1.1.

\section{Background Material}

Let $M_{\ell}$ be a germ at 0 of a smooth Levi non-degenerate hypersurface in $\mathbb{C}^{n+1}$ given in (1). By an easy part of the Chern-Moser normal form theory, after a holomorphic change of coordinates, $M_{\ell}$ near the origin is expressed as

$$
M_{\ell}=\left\{(z, w) \in \mathbb{C}^{n} \times \mathbb{C}: \Im w=|z|_{\ell}^{2}+\frac{1}{4} \mathcal{S}(z)+o(4)\right\} .
$$

Here, for any $n$-tuples $a$ and $b,\langle a, \bar{b}\rangle_{\ell}:=-\sum_{j=1}^{\ell} a_{j} \bar{b}_{j}+\sum_{j=\ell+1}^{n} a_{j} \bar{b}_{j}$ and $|a|_{\ell}^{2}=$ $\langle a, \bar{a}\rangle_{\ell}, \mathcal{S}(z):=\sum_{1 \leq \alpha, \beta, \gamma, \delta \leq n} s_{\alpha \bar{\beta} \gamma \bar{\delta}} z_{\alpha} \bar{z}_{\beta} z_{\gamma} \bar{z}_{\delta}$ is a homogeneous polynomial of bidegree $(2,2)$ satisfying

$$
\begin{aligned}
& s_{\alpha \bar{\beta} \gamma \bar{\delta}}=s_{\gamma \bar{\beta} \alpha \bar{\delta}}=s_{\gamma \bar{\delta} \alpha \bar{\beta}}, \quad \overline{s_{\alpha \bar{\beta} \gamma \bar{\delta}}}=s_{\beta \bar{\alpha} \delta \bar{\gamma}}, \\
& -\sum_{\alpha=1}^{\ell} s_{\alpha \bar{\alpha} \gamma \bar{\delta}}+\sum_{\alpha=\ell+1}^{n} s_{\alpha \bar{\alpha} \gamma \bar{\delta}}=0 .
\end{aligned}
$$


$\mathcal{S}$ is called the Chern-Moser-Weyl curvature function of $M_{\ell}$ at 0 . If $\mathcal{S} \equiv 0$, then 0 is said to be a CR umbilical point.

For a holomorphic function $h(z, w)$, we use $h^{\left(k_{1}, k_{2}\right)}$ to denote the sum of homogeneous terms in its Taylor expansion at 0 whose degrees with respect to $z$ and $w$ are $k_{1}$ and $k_{2}$, respectively. Assign 1 to be the weight of $z$ and 2 to be that of $w$. On the other hand, we denote by $h^{(k)}$ the sum of homogeneous terms of weighted degree $k$ in the Taylor expansion of $h$, and write $o_{w t}(k)$ for terms of weighted degree larger than $k$. To simplify our notation, we also preassign the coefficient of $h$ with negative degrees to be 0 .

Now let $\tilde{M}$ be another germ at 0 of a smooth Levi-non-degenerate hypersurface in $\mathbb{C}^{N+1}$ given by

$$
\tilde{M}_{\ell}=\left\{(\tilde{z}, \tilde{w}) \in \mathbb{C}^{N} \times \mathbb{C}: \Im \tilde{w}=|\tilde{z}|_{\ell}^{2}+\frac{1}{4} \tilde{\mathcal{S}}(\tilde{z})+o(4)\right\} .
$$

Let $F$ be a smooth CR map sending $\left(M_{\ell}, 0\right)$ into $\left(\tilde{M}_{\ell}, 0\right)$. Write

$$
F:=(f, \phi, g)=\left(f_{1}, \ldots, f_{n}, \phi_{1}, \ldots, \phi_{N-n}, g\right) \text {. }
$$

Assume that $F$ is CR transversal at 0 . Then, as in [3, Sect. 2], we can write

$$
\begin{aligned}
& \tilde{z}=\tilde{f}(z, w)=\left(f_{1}(z, w), \ldots, f_{N}(z, w)\right)=\lambda z U+\vec{a} w+O\left(|(z, w)|^{2}\right) \\
& \tilde{w}=g(z, w)=\sigma \lambda^{2} w+O\left(|(z, w)|^{2}\right) .
\end{aligned}
$$

Here, $U$ can be extended to an $N \times N$ matrix $\tilde{U} \in S U(N, \ell)$ (namely, $\langle X \tilde{U}, Y \overline{\tilde{U}}\rangle_{\ell}=$ $\langle X, Y\rangle_{\ell}$ for any $\left.X, Y \in \mathbb{C}^{N}\right), \vec{a} \in \mathbb{C}^{N}$ and $\lambda>0, \sigma= \pm 1$ with $\sigma=1$ for $\ell<\frac{n}{2}$. When $\sigma=-1$, by considering $F \circ \tau_{n / 2}$ instead of $F$, where $\tau_{\frac{n}{2}}\left(z_{1}, \ldots, z_{\frac{n}{2}}, z_{\frac{n}{2}+1}, \ldots\right.$, $\left.z_{n}, w\right)=\left(z_{\frac{n}{2}+1}, \ldots, z_{n}, z_{1}, \ldots, z_{\frac{n}{2}},-w\right)$, we can make $\sigma=1$. Hence, we will assume in what follows that $\sigma=1$.

A result of [15] states that the Chern-Moser-Weyl curvature tensor decreases in the null space of the Levi-form by CR embeddings if $\frac{n}{2}>\ell>0$. When $\ell=\frac{n}{2}$, by choosing an appropriate contact form for the Chern-Moser-Weyl tensor, the same phenomenon also holds. Moreover, as in [15], $F$ can be normalized as follows.

Proposition 2.1 [15] Let $M_{\ell}$ and $\tilde{M}_{\ell}$ be defined by (2) and (3), respectively, and let $F$ be a smooth $C R$ map sending $M_{\ell}$ into $\tilde{M}_{\ell}$ given by (4) and (5) with $\sigma=1$. Then after composing $F$ from the left by some automorphism $T \in \operatorname{Aut}_{0}\left(H_{\ell}^{N+1}\right)$ preserving the origin, the following holds:

$$
\begin{aligned}
& F^{\sharp}=\left(f^{\sharp}, \phi^{\sharp}, g^{\sharp}\right):=T \circ F, \quad \text { with } \\
& f^{\sharp}(z, w)=z+\frac{i}{2} a^{(1,0)}(z) w+o_{w t}(3), \\
& \phi^{\sharp}(z, w)=\phi^{(2,0)}(z)+o_{w t}(2), \\
& g^{\sharp}(z, w)=w+o_{w t}(4),
\end{aligned}
$$


and

$$
\left\langle a^{(1,0)}(z), \bar{z}\right\rangle_{\ell}|z|_{\ell}^{2}=\left|\phi^{(2,0)}(z)\right|^{2}+\frac{1}{4}\left(\mathcal{S}(z)-\lambda^{-2} \tilde{\mathcal{S}}(\lambda(z, 0) \tilde{U})\right) .
$$

In particular, the automorphism $T$ is given by

$$
T(\tilde{z}, \tilde{w})=\frac{\left(\lambda^{-1}\left(\tilde{z}-\lambda^{-2} \vec{a} \tilde{w}\right) \tilde{U}^{-1}, \lambda^{-2} \tilde{w}\right)}{q(\tilde{z}, \tilde{w})},
$$

with $r_{0}=\frac{1}{2} \Re\left\{g_{w w}^{\prime \prime}(0)\right\}, q(\tilde{z}, \tilde{w})=1+2 i\left\langle\tilde{z}, \lambda^{-2} \overline{\vec{a}}\right\rangle_{\ell}+\lambda^{-4}\left(r_{0}-i|\vec{a}|_{\ell}^{2}\right) \tilde{w}$. Moreover, $F^{\sharp}$ sends $M_{\ell}$ into $\tilde{M}^{\sharp}:=T\left(\tilde{M}_{\ell}\right)$ given by

$$
\tilde{M}^{\sharp}=\left\{\left(\tilde{z}^{\sharp}, \tilde{w}^{\sharp}\right) \in \mathbb{C}^{N+1}: \Im \tilde{w}^{\sharp}=\left|\tilde{z}^{\sharp}\right|_{\ell}^{2}+\frac{1}{4} \tilde{\mathcal{S}}^{\sharp}\left(\tilde{z}^{\sharp}\right)+R\left(\tilde{z}^{\sharp}, \overline{\tilde{z}^{\sharp}}, \Re \tilde{w}^{\sharp}\right)\right\},
$$

with $\tilde{\mathcal{S}}^{\sharp}\left(z^{\sharp}\right)=\lambda^{-2} \tilde{\mathcal{S}}\left(\lambda z^{\sharp} \tilde{U}\right)$ and $R\left(\tilde{z}^{\sharp}, \overline{\tilde{z}^{\sharp}}, \Re \tilde{w}^{\sharp}\right)=o(4)$.

\section{Two Preliminary Lemmas}

In this section, we give two lemmas, which will be used in the proof of the main theorem.

For any polynomial $h(z, \bar{z})$, denote by $\|h\|$ the maximum absolute value of all coefficients of terms in $h$.

Lemma 3.1 (1) Let $X(z, \bar{z})$ and $Y(z, \bar{z})$ be two polynomials such that $X(z, \bar{z})=$ $Y(z, \bar{z})|z|_{\ell}^{2}$. Then $\|Y\|$ is bounded by a constant depending only on $\|X\|$ and the degree of $X$.

(2) Let $h(z)$ be a homogeneous holomorphic polynomial of degree $d$ in $z \in \mathbb{C}^{n}$. If $|h(z)| \leq c|z|^{d}$ on $\left\{|z|_{\ell}^{2}=0\right\}$, then $\|h\| \leq C$ for some $C$ depending only on $c$ and $d$.

Proof of Lemma 3.1 (1) Suppose not. Then there is a sequence of polynomials $\left\{X_{j}, Y_{j}\right\}$ with $X_{j}=Y_{j}|z|_{\ell}^{2}$ such that $\left\|Y_{j}\right\|=1, \operatorname{deg}\left(X_{j}\right), \operatorname{deg}\left(Y_{j}\right)$ are bounded by a fixed constant but $\left\|X_{j}\right\| \rightarrow 0$. By passing to a subsequence, we can assume that $X_{j} \rightarrow 0, Y_{j} \rightarrow Y$ with $\|Y\|=1$ and $0=Y|z|_{\ell}^{2}$. It then follows that $Y \equiv 0$. This is a contradiction.

(2) For any point $z^{0} \in \mathbb{C}^{n}$ with $\left|z^{0}\right|_{\ell}^{2} \neq 0$, suppose without loss of generality that $\sum_{j=1}^{\ell}\left|z_{j}^{0}\right|^{2}>\sum_{j=\ell+1}^{n}\left|z_{j}^{0}\right|^{2}$. Consider the closed subset given by $P:=\left\{z \in \mathbb{C}^{n}\right.$ : $\left.z_{j}=z_{j}^{0}, 1 \leq j \leq \ell, \sum_{j=\ell+1}^{n}\left|z_{j}\right|^{2} \leq \sum_{j=1}^{\ell}\left|z_{j}^{0}\right|^{2}\right\}$. Then $\partial P \subset\left\{|z|_{\ell}^{2}=0\right\}$ and $z^{0} \in P$. Hence, $\left|h\left(z^{0}\right)\right| \leq \sup _{z \in \partial P}|h(z)| \leq c \sup _{z \in\left\{\left\{|z|_{\ell}^{2}=0\right\} \cap P\right\}}|z|^{d} \leq 2^{d / 2} c\left|z^{0}\right|^{d}$ by the maximum principle. Therefore, $\|h\| \leq C$ for some $C$ depending only on $c$ and $d$.

Lemma 3.2 Let $f_{1}$ and $f_{2}$ be two homogeneous holomorphic polynomials of degree $d$ and degree 2 , respectively. Assume $\left\|f_{2}\right\| \geq c$ and

$$
f_{1}(z) f_{2}(\bar{z})=H(z, \bar{z}) \bmod \left(|z|_{\ell}^{2}\right)
$$


for some homogeneous polynomial $H$ of degree $d+2$ with $\|H\| \leq c^{\prime}$. Then $\left\|f_{1}\right\| \leq C$ for some $C$ depending only on $c, c^{\prime}$, and $d$.

Proof of Lemma 3.2 Without loss of generality, we assume that $\left\|f_{2}\right\|=1$ and thus $c^{\prime}$ is replaced by $c^{\prime} / c$.

First, we assume that the absolute value of the coefficient of the term $z_{l}^{2}$ in $f_{2}$ is greater than or equal to $\frac{1}{16}$. For simplicity of notation, assume $l=1$. Complexifying (6), we get

$$
f_{1}(z) f_{2}(\chi)=H(z, \chi) \bmod \left(\langle z, \chi\rangle_{\ell}\right) .
$$

Write $z=\left(z_{1}, z^{\prime}\right) \in \mathbb{C} \times \mathbb{C}^{n-1}, \chi=\left(\chi_{1}, \chi^{\prime}\right) \in \mathbb{C} \times \mathbb{C}^{n-1}$, and $u:=\langle z, \chi\rangle_{\ell}$. Letting $\chi_{1}=1$, then we have $z_{1}=\left\langle z^{\prime}, \chi^{\prime}\right\rangle_{\ell-1}-u$, and the above equation becomes

$$
f_{1}\left(\left\langle z^{\prime}, \chi^{\prime}\right\rangle_{\ell-1}-u, z^{\prime}\right) f_{2}\left(1, \chi^{\prime}\right)=H\left(\left\langle z^{\prime}, \chi^{\prime}\right\rangle_{\ell-1}-u, z^{\prime}, 1, \chi^{\prime}\right) \bmod (u) .
$$

Letting $u=0$ in the above expression, we get

$$
f_{1}\left(\left\langle z^{\prime}, \chi^{\prime}\right\rangle_{\ell-1}, z^{\prime}\right) f_{2}\left(1, \chi^{\prime}\right)=H\left(\left\langle z^{\prime}, \chi^{\prime}\right\rangle_{\ell-1}, z^{\prime}, 1, \chi^{\prime}\right)=: \tilde{H}\left(z^{\prime}, \chi^{\prime}\right),
$$

for some polynomial $\tilde{H}$ with coefficients bounded depending only on $c, c^{\prime}$. On the other hand, by the assumption on $f_{2}$, there exists some small number $\epsilon>0$, depending only on $c$ and $c^{\prime}$, such that $\left|f_{2}\left(1, \chi^{\prime}\right)\right| \geq \frac{1}{32}$ as $\left|\chi^{\prime}\right| \leq \epsilon$. Therefore, the above equation implies $\left|f_{1}\left(\left\langle z^{\prime}, \chi^{\prime}\right\rangle_{\ell-1}, z^{\prime}\right)\right|$ is bounded by some constant depending only on $c, c^{\prime}, d$ when $\left|\chi^{\prime}\right| \leq \epsilon$ and $\left|z^{\prime}\right| \leq 1$. Now write $h\left(\chi^{\prime}, z^{\prime}\right):=f_{1}\left(\left\langle z^{\prime}, \chi^{\prime}\right\rangle_{\ell-1}, z^{\prime}\right)$. Applying the Cauchy estimates to $h$, we get $\|h\|$ is bounded by some constant depending only on $c, c^{\prime}$, and $d$. By tracing the coefficients of $f_{1}\left(z_{1}, z^{\prime}\right)$ via those of $h$, we obtain the boundedness of $\left\|f_{1}\right\|$ by some constant depending only on $c, c^{\prime}$, and $d$.

Next, suppose that the coefficient of $z_{1}^{2}$ in $f_{1}$ has absolute value less than $\frac{1}{16}$. By making a linear change of coordinates which preserves the quadric form $-\sum_{j=1}^{\ell}\left|z_{j}\right|^{2}+\sum_{j=\ell+1}^{n}\left|z_{j}\right|^{2}$, we can always make the coefficient of the term $z_{l}^{2}$ for some $l$ in $f_{2}$ to have absolute value bigger than $\frac{1}{16}$ (after normalizing $\left\|f_{2}\right\|=1$ ) and thus reduce the situation to what we did above.

Indeed, suppose that the absolute value of the coefficients of $z_{k}^{2}, 1 \leq k \leq n$ are all less than $\frac{1}{16}$ and that of $z_{j} z_{l}$ is 1 instead. Suppose that $j, l \leq \ell$ or $j, l>\ell$. Applying coordinates change: $z_{j}=\frac{1}{\sqrt{2}}\left(z_{j}^{\prime}+z_{l}^{\prime}\right), z_{l}=\frac{1}{\sqrt{2}}\left(z_{l}^{\prime}-z_{j}^{\prime}\right)$, and $z_{k}=z_{k}^{\prime}$ for $k \neq j$, $l$, we then see that the absolute value of the coefficients of both $\left(z_{l}^{\prime}\right)^{2}$ and $\left(z_{j}^{\prime}\right)^{2}$ in $f_{2}$ are greater than or equal to $\frac{1}{4}$ and $\left\|f_{2}\right\| \leq 2$ in the new coordinate system. Hence, when we normalize $\left\|f_{2}\right\|=1$, we see the coefficient of $\left(z_{j}^{\prime}\right)^{2}$ has absolute value at least $\frac{1}{16}$. Suppose that $j \leq \ell$ and $l>\ell$. We can then define $z_{j}=\sqrt{2} z_{j}^{\prime}-z_{l}^{\prime}, z_{l}=z_{j}^{\prime}-\sqrt{2} z_{l}^{\prime}$, and $z_{k}=z_{k}^{\prime}$ for $k \neq j, l$. In the new coordinate system, $\left\|f_{2}\right\| \leq 3$, but the coefficient of $\left(z_{j}^{\prime}\right)^{2}$ has absolute value at least $\frac{1}{4}$. We are thus similarly done in this case, too.

Remark 3.3 With a similar argument, one sees that Lemma 3.2 still holds when $f_{2}$ has degree $k \geq 2$. In this setting, $C$ depends only on $c, c^{\prime}, d$, and $k$. 


\section{Proof of the Main Theorem}

We give a proof of our theorem in this section, which is also partially based on the rescaling method.

Proof of Theorem 1.1 Seeking a contradiction, suppose that $F$ is neither CR transversal to $M_{\ell}$ at 0 nor sends $U$ into $H_{\ell}^{n+2}$. Since the set of points where the CR transversality holds for $F$ forms an open dense subset in $M_{\ell}$ by a result in [2], we can pick a sequence $\left\{p_{j}\right\} \in M_{\ell}$ such that $\left\{p_{j}\right\}$ approaches 0 and $F$ is CR transversal at $p_{j}$ with $j \geq 1$. Write $q_{j}:=F\left(p_{j}\right)$. Now at each $p_{j}$, applying the normalization process to $F$ as mentioned in Proposition 2.1 (with $N=n+1$ ), we have the following:

$$
\begin{aligned}
& F_{p_{j}}^{\sharp}=\left(f_{p_{j}}^{\sharp}, \phi_{p_{j}}^{\sharp}, g_{p_{j}}^{\sharp}\right) \\
& =\left(f_{1} \underset{p_{j}}{\sharp}, \ldots, f_{n} \underset{p_{j}}{\sharp}, \phi_{p_{j}}^{\sharp}, g_{p_{j}}^{\sharp}\right):=T_{p_{j}} \circ \tau_{F\left(p_{j}\right)} \circ F \circ \sigma_{p_{j}} \text {, where } \\
& f_{p_{j}}^{\sharp}(z, w)=z+\frac{i}{2} a_{p_{j}}^{(1,0)}(z) w+o_{w t}(3), \\
& \phi_{p_{j}}^{\sharp}(z, w)=\phi_{p_{j}}^{(2,0)}(z)+o_{w t}(2), \\
& g_{p_{j}}^{\sharp}(z, w)=w+o_{w t}(4),
\end{aligned}
$$

with the CR version of the Gauss-Codazzi equation

$$
\left\langle a_{p_{j}}^{(1,0)}(z), \bar{z}\right\rangle_{\ell}|z|_{\ell}^{2}=\left|\phi_{p_{j}}^{(2,0)}(z)\right|^{2}+\frac{1}{4} \mathcal{S}_{p_{j}}(z)
$$

Here $\tau_{F\left(p_{j}\right)}$ is the translation of $H_{\ell}^{n+2}$ sending $F\left(p_{j}\right)$ to $0, \sigma_{p_{j}}$ is a biholomorphic map sending 0 to $p_{j}$, and $\sigma_{p_{j}}^{-1}\left(M_{\ell}\right)$ is in the normal form up to the fourth order. We can make $\sigma_{p_{j}}$ depend smoothly on $p_{j}$. Also, write $\mathcal{S}_{p_{j}}$ for the resulting ChernMoser-Weyl curvature function of $M_{\ell}$ at $p_{j}$. By making use of the fact that $F$ is not CR transversal at 0 , we get that $\lim _{j \rightarrow \infty} \lambda_{p_{j}}=0$ for $\lambda_{p_{j}}$ defined in (5) with $F$ replaced by $\tau_{F\left(p_{j}\right)} \circ F \circ \sigma_{p_{j}}$. Now at each point $p_{j}, F_{p_{j}}^{\sharp}$ given in (7) sends $\sigma_{p_{j}}^{-1}\left(M_{\ell}\right)$ into $H_{\ell}^{n+2}$. We then have for $(z, u) \approx 0$,

$$
\begin{aligned}
& -\Im g_{p_{j}}^{\sharp}\left(z, u+i\left(|z|_{\ell}^{2}+o_{w t}(3)\right)\right)+\left|f_{p_{j}}^{\sharp}\left(z, u+i\left(|z|_{\ell}^{2}+o_{w t}(3)\right)\right)\right|_{\ell}^{2} \\
& \quad+\left|\phi_{p_{j}}^{\sharp}\left(z, u+i\left(|z|_{\ell}^{2}+o_{w t}(3)\right)\right)\right|^{2}=0 .
\end{aligned}
$$

Here, $\left(z, u+i\left(|z|_{\ell}^{2}+o_{w t}(3)\right)\right)$ is a local parameterization of $\sigma_{p_{j}}^{-1}\left(M_{\ell}\right)$ near 0 . Due to the smooth dependence of $\sigma_{p_{j}}$ with respect to $p_{j}$, the error term $o_{w t}(3)$ depends smoothly on $p_{j}$.

In the sequel, for simplicity of notation, we will drop $\sharp$ and write $F_{p_{j}}$ for $F_{p_{j}}^{\sharp}$. We will also abuse our notation and use $C_{1}, C_{2}$, or $C$ to denote positive constants independent of $p$, and use $H(\cdot)$ to denote a (real analytic) polynomial function with $\|H\|$ bounded by a constant independent of $p$. All these quantities may be different in different contexts. We will divide our proof into several steps. 
Step 1: We prove in this step the estimate: $C_{2} \leq\left\|\phi_{p_{j}}^{(2,0)}\right\| \leq C_{1}$.

Assume that $\left|\mathcal{S}_{p_{j}}(z)\right| \leq c_{1}|z|^{4}$ for some positive number $c_{1}$ independent of $p_{j}$. Restricting the Gauss equation (8) on the Levi-cone $\left\{|z|_{\ell}^{2}=0\right\}$, we have

$$
4\left|\phi_{p_{j}}^{(2,0)}(z)\right|^{2}=-\mathcal{S}_{p_{j}}(z)
$$

Since $\left\{\mathcal{S}_{p_{j}}\right\}_{j=1}^{\infty}$ is uniformly bounded, this then forces $\phi_{p_{j}}^{(2,0)}$ to be uniformly bounded on $\left\{|z|_{\ell}^{2}=0\right\}$ for all $p_{j}$ 's. By Lemma 3.1, $\left\|\phi_{p_{j}}^{(2,0)}\right\| \leq C_{1}$. It also follows that $\left\|a_{p_{j}}^{(1,0)}\right\| \leq C_{1}$. On the other hand, since $0 \in M_{\ell}$ is not CR umbilical, $\mathcal{S}_{0} \not \equiv 0$. Since $\mathcal{S}_{0}$ is not divisible by $|z|_{\ell}^{2}$, we have $\sup _{\left\{|z|_{\ell}^{2}=0\right\} \cap\left\{|z|^{2}=1\right\}}\left|S_{0}(z)\right|>c$ for some $c>0$. By the smoothness of $\mathcal{S}$ in terms of $p_{j}, \sup _{\left\{|z|_{\ell}^{2}=0\right\} \cap\left\{|z|^{2}=1\right\}}\left|S_{p_{j}}(z)\right|>\frac{c}{2}$ for all $p_{j}$ after passing to a subsequence. It therefore follows from (10) that $\sup _{\left\{|z|_{\ell}^{2}=0\right\} \cap\left\{|z|^{2}=1\right\}}\left|\phi_{p_{j}}^{(2,0)}(z)\right|>\sqrt{\frac{c}{2}}$, and hence $\left\|\phi_{p_{j}}^{(2,0)}\right\| \geq C_{2}$.

Step 2: For each fixed $k \geq 1$, we next claim that there exists some positive number $C$ such that

$$
\begin{aligned}
& \left\|\phi_{p}^{(k)}(\cdot, 1)\right\| \leq C, \\
& \left\|f_{p}^{(k+1)}(\cdot, 1)\right\| \leq C, \\
& \left\|g_{p}^{(k+2)}(\cdot, 1)\right\| \leq C,
\end{aligned}
$$

for any $p \in\left\{p_{j}\right\}_{j=1}^{\infty}$. Here, for a holomorphic function $h(z, w)$, we define $h^{(k)}(z, 1):=$ $\left.\left(h^{(k)}(z, w)\right)\right|_{w=1}$.

The easy case with $k=1$ or 2 is already done in Step 1 and (7). Now assume (11), (12), and (13) hold for $k \leq 2 m-1$ and $k \leq 2 m$ ( $m \geq 1)$. Namely, assume that $\left\|g_{p}^{(r)}\right\|_{0 \leq r \leq 2 m+2},\left\|f_{p}^{(r)}\right\|_{0 \leq r \leq 2 m+1}$ and $\left\|\phi_{p}^{(r)}\right\|_{0 \leq r \leq 2 m}$ are bounded by some constant independent of $p$. We need to show inductively that (11), (12), and (13) also hold for $k=2 m+1$ and $k=2 m+2$.

Collect terms of weighted degree $k+2$ in the Taylor expansion of (9). We have the following:

$$
\begin{aligned}
- & \Im g_{p}^{(k+2)}\left(z, u+i|z|_{\ell}^{2}\right)+2 \Re\left\langle f_{p}^{(k+1)}\left(z, u+i|z|_{\ell}^{2}\right),\left.\bar{z}\right|_{\ell} ^{2}\right. \\
& +2 \Re \phi_{p}^{(k)}\left(z, u+i|z|_{\ell}^{2}\right) \cdot \overline{\phi_{p}^{(2,0)}(z)} \\
= & H\left(\left.g_{p}^{(r)}\right|_{0 \leq r \leq k+1},\left.f_{p}^{(r)}\right|_{0 \leq r \leq k},\left.\phi_{p}^{(r)}\right|_{0 \leq r \leq k-1}\right) .
\end{aligned}
$$

Case 1: $k=2 m+1$. Collecting terms in (14) of degree 1 in $z$ and degree $m+1$ in $u$, we obtain

$$
-\frac{1}{2 i} g_{p}^{(1, m+1)}(z, 1) u^{m+1}+\left\langle z, \overline{f_{p}^{(0, m+1)}(z, 1)}\right\rangle_{\ell} u^{m+1}=H(z, \bar{z}) u^{m+1} .
$$


Collecting terms in (14) of degree 2 in $z$, degree 1 in $\bar{z}$ and degree $m$ in $u$, we obtain

$$
\begin{aligned}
& -\frac{1}{2 i}(m+1) g_{p}^{(1, m+1)}(z, 1) u^{m}\left(i|z|_{\ell}^{2}\right)+(m+1)\left\langle z, \overline{f_{p}^{(0, m+1)}(z, 1) i|z|_{\ell}^{2}}\right\rangle_{\ell} u^{m} \\
& \quad+\left\langle f_{p}^{(2, m)}(z, 1) u^{m}, \bar{z}\right\rangle_{\ell}+\phi_{p}^{(2,0)}(z) \cdot \overline{\phi_{p}^{(1, m)}(z, 1)} u^{m}=H(z, \bar{z}) u^{m} .
\end{aligned}
$$

Collecting terms in (14) of degree 3 in $z$, degree 2 in $\bar{z}$ and degree $m-1$ in $u$, we obtain

$$
\begin{aligned}
& -\frac{1}{2 i} C_{m+1}^{2} g_{p}^{(1, m+1)}(z, 1) u^{m-1}\left(i|z|_{\ell}^{2}\right)^{2}+C_{m+1}^{2}\left\langle z \overline{f_{p}^{(0, m+1)}(z, 1)\left(i|z|_{\ell}^{2}\right)^{2}}\right\rangle_{\ell} u^{m-1} \\
& \quad+m\left\langle f_{p}^{(2, m)}(z, 1)\left(i|z|_{\ell}^{2}\right) u^{m-1}, \bar{z}\right\rangle_{\ell}+m \phi_{p}^{(2,0)}(z) \cdot \overline{\phi_{p}^{(1, m)}(z, 1) i|z|_{\ell}^{2}} u^{m-1} \\
& \quad+\overline{\phi_{p}^{(2,0)}(z)} \cdot \phi_{p}^{(3, m-1)}(z, 1) u^{m-1}=H(z, \bar{z}) u^{m-1}
\end{aligned}
$$

Restricting (17) on $\left\{|z|_{\ell}^{2}=0\right\}$ and applying Lemma 3.2, we get

$$
\left\|\phi_{p}^{(3, m-1)}(\cdot, 1)\right\| \leq C .
$$

Combining (18), (15), and (17), and applying Lemma 3.1, we get

$$
\left\langle f_{p}^{(2, m)}(z, 1)\left(i|z|_{\ell}^{2}\right), \bar{z}\right\rangle_{\ell}=-\phi_{p}^{(2,0)}(z) \cdot \overline{\phi_{p}^{(1, m)}(z, 1) i|z|_{\ell}^{2}}+H(z, \bar{z}),
$$

or equivalently,

$$
\left\langle f_{p}^{(2, m)}(z, 1), \bar{z}\right\rangle_{\ell}=\phi_{p}^{(2,0)}(z) \cdot \overline{\phi_{p}^{(1, m)}(z, 1)}+H(z, \bar{z}) .
$$

Substituting (19) into (16), we have after simplification

$$
\begin{aligned}
& -\frac{1}{2 i}(m+1) g_{p}^{(1, m+1)}(z, 1)\left(i|z|_{\ell}^{2}\right)+(m+1)\left\langle z \overline{f_{p}^{(0, m+1)}(z, 1) i|z|_{\ell}^{2}}\right\rangle_{\ell} \\
& \quad+2 \phi_{p}^{(2,0)}(z) \cdot \overline{\phi_{p}^{(1, m)}(z, 1)}=H(z, \bar{z}) .
\end{aligned}
$$

Restricting (20) on $\left\{|z|_{\ell}^{2}=0\right\}$ and applying Lemma 3.2 again, we get

$$
\left\|\phi_{p}^{(1, m)}(\cdot, 1)\right\| \leq C .
$$

Hence from (19), we get

$$
\left\|f_{p}^{(2, m)}(\cdot, 1)\right\| \leq C .
$$

Plug (21) into (20). We then have after simplification, together with Lemma 3.1,

$$
-\frac{1}{2 i} g_{p}^{(1, m+1)}(z, 1)-\left\langle z, \overline{f_{p}^{(0, m+1)}(z, 1)}\right\rangle_{\ell}=H(z, \bar{z}) .
$$

Combining (22) and (15), we get

$$
\left\|g_{p}^{(1, m+1)}(\cdot, 1)\right\| \leq C,
$$




$$
\left\|f_{p}^{(0, m+1)}(\cdot, 1)\right\| \leq C .
$$

Next, to estimate $\left\|g_{p}^{(2 m+3-2 r, r)}(\cdot, 1)\right\|, \quad\left\|f_{p}^{(2 m+4-2 r, r-1)}(\cdot, 1)\right\|, \quad$ and $\left\|\phi_{p}^{(2 m+5-2 r, r-2)}(\cdot, 1)\right\|(0 \leq r \leq m)$, we first collect terms in (14) of degree $2 m+$ $3-2 r(\geq 3)$ in $z$ and degree $r$ in $u$ to get

$$
\left\|g_{p}^{(2 m+3-2 r, r)}(\cdot, 1)\right\| \leq C .
$$

(Notice that by convention, the coefficients of terms with negative degrees are defined to be 0 .)

Collecting terms in (14) of degree $2 m+4-2 r(\geq 4)$ in $z$, degree 1 in $\bar{z}$, and degree $r-1$ in $u$, we obtain

$$
-\frac{1}{2 i} r g_{p}^{(2 m+3-2 r, r)}(z, 1) u^{r-1}\left(i|z|_{\ell}^{2}\right)+\left\langle f_{p}^{(2 m+4-2 r, r-1)}(z, 1) u^{r-1}, \bar{z}\right\rangle_{\ell}=H(z, \bar{z}) u^{r-1} .
$$

Substituting (23) into the above, we have

$$
\left\|f_{p}^{(2 m+4-2 r, r-1)}(\cdot, 1)\right\| \leq C .
$$

Collecting terms in (14) of degree $2 m+5-2 r(\geq 5)$ in $z$, degree 2 in $\bar{z}$, and degree $r-2$ in $u$, we obtain

$$
\begin{aligned}
& -\frac{1}{2 i} C_{r}^{2} g_{p}^{(2 m+3-2 r, r)}(z, 1) u^{r-2}\left(i|z|_{\ell}^{2}\right)^{2}+(r-1)\left\langle f_{p}^{(2 m+4-2 r, r-1)}(z, 1)\left(i|z|_{\ell}^{2} u^{r-2}, \bar{z}\right\rangle_{\ell}\right. \\
& \quad+\phi_{p}^{(2 m+5-2 r, r-2)}(z, 1) u^{r-2} \cdot \overline{\phi_{p}^{(2,0)}(z)}=H(z, \bar{z}) u^{r-2} .
\end{aligned}
$$

Substituting (23) and (4) into the above, we have

$$
\left\|\phi_{p}^{(2 m+5-2 r, r-2)}(\cdot, 1)\right\| \leq C .
$$

Case 2: $k=2 m+2$. Collecting terms in (14) of degree $m+2$ in $u$, we get

$$
-\frac{1}{2 i}\left(g_{p}^{(0, m+2)}(z, 1)-\overline{g^{(0, m+2)}(z, 1)}\right) u^{m+2}=H(z, \bar{z}) u^{m+2} .
$$

We thus have

$$
\left\|\Im g_{p}^{(0, m+2)}(\cdot, 1)\right\| \leq C .
$$

Collecting terms in (14) of degree 1 in $z$, degree 1 in $\bar{z}$, and degree $m+1$ in $u$, we have

$$
\begin{aligned}
& -\frac{m+2}{2 i}\left(g_{p}^{(0, m+2)}(z, 1)\left(i|z|_{\ell}^{2}\right)-\overline{g^{(0, m+2)}(z, 1)\left(i|z|_{\ell}^{2}\right)}\right) u^{m+1} \\
& \quad+2 \Re\left\langle f_{p}^{(1, m+1)}(z, 1), \bar{z}\right\rangle_{\ell} u^{m+1}=H(z, \bar{z}) u^{m+1} .
\end{aligned}
$$

We thus get

$$
i(m+2) \Re g_{p}^{(0, m+2)}(z, 1)\left(i|z|_{\ell}^{2}\right)+2 \Re\left\langle f_{p}^{(1, m+1)}(z, 1), \bar{z}\right\rangle_{\ell}=H(z, \bar{z}) .
$$


Collect terms in (14) of degree 2 in $z$, degree 2 in $\bar{z}$ and degree $m$ in $u$, we have

$$
\begin{aligned}
& -\frac{1}{2 i} C_{m+2}^{2}\left(g_{p}^{(0, m+2)}(z, 1)-\overline{g^{(0, m+2)}(z, 1)}\right)\left(i|z|_{\ell}^{2}\right)^{2} u^{m} \\
& \quad+2(m+1) \Re\left\langle f_{p}^{(1, m+1)}(z, 1)\left(i|z|_{\ell}^{2}\right), \bar{z}\right\rangle_{\ell} u^{m} \\
& \quad+2 \Re\left(\phi_{p}^{(2, m)}(z, 1) u^{m} \cdot \overline{\phi_{p}^{(2,0)}(z)}\right)=H(z, \bar{z}) u^{m},
\end{aligned}
$$

or equivalently,

$$
\begin{aligned}
& -C_{m+2}^{2} \Im g_{p}^{(0, m+2)}(z, 1)\left(i|z|_{\ell}^{2}\right)^{2}+2(m+1) i \Im\left\langle f_{p}^{(1, m+1)}(z, 1), \bar{z}\right\rangle_{\ell}\left(i|z|_{\ell}^{2}\right) \\
& +2 \Re\left(\phi_{p}^{(2, m)}(z, 1) \cdot \overline{\phi_{p}^{(2,0)}(z)}\right)=H(z, \bar{z}) .
\end{aligned}
$$

Collecting terms in (14) of degree 3 in $z$, degree 3 in $\bar{z}$, and degree $m-1$ in $u$, we have

$$
\begin{aligned}
- & \frac{1}{2 i} C_{m+2}^{3}\left(g_{p}^{(0, m+2)}(z, 1)\left(i|z|_{\ell}^{2}\right)^{3}-\overline{g^{(0, m+2)}(z, 1)\left(i|z|_{\ell}^{2}\right)^{3}}\right) u^{m-1} \\
& +2 C_{m+1}^{2} \Re\left\langle f_{p}^{(1, m+1)}(z, 1)\left(i|z|_{\ell}^{2}\right)^{2}, \bar{z}\right\rangle_{\ell} u^{m-1} \\
& +2 m \Re\left(\phi_{p}^{(2, m)}(z, 1)\left(i|z|_{\ell}^{2}\right) u^{m-1} \cdot \overline{\phi_{p}^{(2,0)}(z)}\right)=H(z, \bar{z}) u^{m-1},
\end{aligned}
$$

or equivalently,

$$
\begin{aligned}
& i C_{m+2}^{3} \Re g_{p}^{(0, m+2)}(z, 1)\left(i|z|_{\ell}^{2}\right)^{2}+2 C_{m+1}^{2} \Re\left\langle f_{p}^{(1, m+1)}(z, 1), \bar{z}\right\rangle_{\ell}\left(i|z|_{\ell}^{2}\right) \\
& \quad+2 m i \Im\left(\phi_{p}^{(2, m)}(z, 1) \cdot \overline{\phi_{p}^{(2,0)}(z)}\right)=H(z, \bar{z}) .
\end{aligned}
$$

Dividing (27) by $m$ and adding it to (26), we have

$$
\phi_{p}^{(2, m)}(z, 1) \cdot \overline{\phi_{p}^{(2,0)}(z)}=H(z, \bar{z}) \bmod \left(|z|_{\ell}^{2}\right) .
$$

By Lemma 3.2, we get

$$
\left\|\phi_{p}^{(2, m)}(\cdot, 1)\right\| \leq C .
$$

Combining (28) with (26) and (27), we have after simplification the following:

$$
\begin{aligned}
& -C_{m+2}^{2} \Im g_{p}^{(0, m+2)}(z, 1)\left(i|z|_{\ell}^{2}\right)+2(m+1) i \Im\left\langle f_{p}^{(1, m+1)}(z, 1), \bar{z}\right\rangle_{\ell}=H(z, \bar{z}), \\
& i C_{m+2}^{3} \Re g_{p}^{(0, m+2)}(z, 1)\left(i|z|_{\ell}^{2}\right)+2 C_{m+1}^{2} \Re\left\langle f_{p}^{(1, m+1)}(z, 1), \bar{z}\right\rangle_{\ell}=H(z, \bar{z}) .
\end{aligned}
$$

Combining (29) with (24) and (25), we get

$$
\begin{aligned}
& \left\|\Re g_{p}^{(0, m+2)}(\cdot, 1)\right\| \leq C, \\
& \left\langle f_{p}^{(1, m+1)}(z, 1), \bar{z}\right\rangle_{\ell}=H(z, \bar{z}) .
\end{aligned}
$$


Therefore, together with (24) again, we obtain

$$
\left\|g_{p}^{(0, m+2)}(\cdot, 1)\right\| \leq C, \quad\left\|f_{p}^{(1, m+1)}(\cdot, 1)\right\| \leq C .
$$

To estimate $\left\|g_{p}^{(2, m+1)}(\cdot, 1)\right\|,\left\|f_{p}^{(3, m)}(\cdot, 1)\right\|,\left\|\phi_{p}^{(0, m+1)}(\cdot, 1)\right\|$, and $\left\|\phi_{p}^{(4, m-1)}(\cdot, 1)\right\|$, we collect terms in (14) of degree 2 in $z$ and degree $m+1$ in $u$ to obtain

$$
-\frac{1}{2 i} g_{p}^{(2, m+1)}(z, 1) u^{m+1}+\phi_{p}^{(2,0)}(z) \cdot \overline{\phi_{p}^{(0, m+1)}(z, 1)} u^{m+1}=H(z, \bar{z}) u^{m+1} .
$$

Collecting terms in (14) of degree 3 in $z$, degree 1 in $\bar{z}$, and degree $m$ in $u$, we obtain

$$
\begin{aligned}
& -\frac{m+1}{2 i} g_{p}^{(2, m+1)}(z, 1) u^{m}\left(i|z|_{\ell}^{2}\right)+(m+1) \phi_{p}^{(2,0)}(z) \cdot \overline{\phi_{p}^{(0, m+1)}(z, 1) i|z|_{\ell}^{2}} u^{m} \\
& \quad+\left\langle f_{p}^{(3, m)}(z, 1) u^{m}, \bar{z}\right\rangle_{\ell}=H(z, \bar{z}) u^{m} .
\end{aligned}
$$

Collecting terms in (14) of degree 4 in $z$, degree 2 in $\bar{z}$, and degree $m-1$ in $u$, we obtain

$$
\begin{aligned}
& -\frac{1}{2 i} C_{m+1}^{2} g_{p}^{(2, m+1)}(z, 1) u^{m-1}\left(i|z|_{\ell}^{2}\right)^{2}+C_{m+1}^{2} \phi_{p}^{(2,0)}(z) \cdot \overline{\phi_{p}^{(0, m+1)}(z, 1)\left(i|z|_{\ell}^{2}\right)^{2}} u^{m-1} \\
& \quad+m\left\langle f_{p}^{(3, m)}(z, 1)\left(i|z|_{\ell}^{2}\right) u^{m-1}, \bar{z}\right\rangle_{\ell}+\phi_{p}^{(4, m-1)}(z, 1) u^{m-1} \cdot \overline{\phi_{p}^{(2,0)}(z)} \\
& =H(z, \bar{z}) u^{m-1} .
\end{aligned}
$$

Restricting (32) on $\left\{|z|_{\ell}^{2}=0\right\}$ and applying Lemma 3.2, we get

$$
\left\|\phi_{p}^{(4, m-1)}(\cdot, 1)\right\| \leq C .
$$

Therefore, substituting (33) into (32) and applying Lemma 3.1, we have

$$
\begin{aligned}
& C_{m+1}^{2}\left(i|z|_{\ell}^{2}\right)\left(-\frac{1}{2 i} g_{p}^{(2, m+1)}(z, 1)+\phi_{p}^{(2,0)}(z) \cdot \overline{\phi_{p}^{(0, m+1)}(z, 1)}\right)+m\left\langle f_{p}^{(3, m)}(z, 1), \bar{z}\right\rangle_{\ell} \\
& \quad=H(z, \bar{z}) .
\end{aligned}
$$

Substituting (30) into (34), we get

$$
\left\|f_{p}^{(3, m)}(\cdot, 1)\right\| \leq C .
$$

Then (31) gives

$$
-\frac{1}{2 i} g_{p}^{(2, m+1)}(z, 1)-\phi_{p}^{(2,0)}(z) \cdot \overline{\phi_{p}^{(0, m+1)}(z, 1)}=H(z, \bar{z}) .
$$

(35) and (30) together give

$$
\begin{gathered}
\left\|g_{p}^{(2, m+1)}(\cdot, 1)\right\| \leq C, \\
\left\|\phi_{p}^{(0, m+1)}(\cdot, 1)\right\| \leq C .
\end{gathered}
$$


For general $\left\|g_{p}^{(2 m+4-2 r, r)}(\cdot, 1)\right\|,\left\|f_{p}^{(2 m+5-2 r, r-1)}(\cdot, 1)\right\|,\left\|\phi_{p}^{(2 m+6-2 r, r-2)}(\cdot, 1)\right\|(0 \leq$ $r \leq m$ ), we collect terms in (14) of degree $2 m+4-2 r(\geq 4)$ in $z$ and degree $r$ in $u$ and get

$$
\left\|g_{p}^{(2 m+4-2 r, r)}(\cdot, 1)\right\| \leq C .
$$

Collecting terms in (14) of degree $2 m+5-2 r(\geq 5)$ in $z$, degree 1 in $\bar{z}$, and degree $r-1$ in $u$, then we obtain

$$
-\frac{1}{2 i} r g_{p}^{(2 m+4-2 r, r)}(z, 1) u^{r-1}\left(i|z|_{\ell}^{2}\right)+\left\langle f_{p}^{(2 m+5-2 r, r-1)}(z, 1) u^{r-1}, \bar{z}\right\rangle_{\ell}=H(z, \bar{z}) .
$$

Substituting (36) into the above, we have

$$
\left\|f_{p}^{(2 m+5-2 r, r-1)}(\cdot, 1)\right\| \leq C .
$$

Collecting terms in (14) of degree $2 m+6-2 r$ in $z$, degree 2 in $\bar{z}$, and degree $r-2$ in $u$, we obtain

$$
\begin{aligned}
& -\frac{1}{2 i} C_{r}^{2} g_{p}^{(2 m+4-2 r, r)}(z, 1) u^{r-2}\left(i|z|_{\ell}^{2}\right)^{2}+(r-1)\left\langle f_{p}^{(2 m+5-2 r, r-1)}(z, 1)\left(i|z|_{\ell}^{2} u^{r-2}, \bar{z}\right\rangle_{\ell}\right. \\
& \quad+\phi_{p}^{(2 m+6-2 r, r-2)}(z, 1) u^{r-2} \cdot \overline{\phi_{p}^{(2,0)}(z)}=H(z, \bar{z}) .
\end{aligned}
$$

Substituting (36) and (37) into the above, we have

$$
\left\|\phi_{p}^{(2 m+6-2 r, r-2)}(\cdot, 1)\right\| \leq C .
$$

This completes the induction.

Step 3: We have now shown that for each fixed $k,\left\{\left\|F_{p_{j}}^{(k)}\right\|\right\}_{j=1}^{\infty}$ is bounded by some constant independent of $j$. In particular, since $\left\{\left\|F_{p_{j}}^{(2)}\right\|\right\}_{j=1}^{\infty}$ is bounded, we can find a subsequence $\left\{p_{j}^{(2)}\right\}_{j=1}^{\infty}$ of $\left\{p_{j}\right\}_{j=1}^{\infty}$ such that $\left\{F_{p_{j}^{(2)}}^{(2)}\right\}_{j=1}^{\infty}$ converges on compacta as $j \rightarrow \infty$. Similarly, we find inductively sequences $\left\{p_{j}^{(k)}\right\}_{j=1}^{\infty} \subset\left\{p_{j}^{(k-1)}\right\}_{j=1}^{\infty}$ such that $\left\{F_{p_{j}(k)}^{(k)}\right\}_{j=1}^{\infty}$ converges. Pick the diagonal subsequence $\left\{p_{j}^{(j)}\right\}_{j=1}^{\infty}$ and denote it still as $\left\{p_{j}\right\}_{j=1}^{\infty}$. Then for each $k,\left\{F_{p_{j}}^{(k)}\right\}_{j=1}^{\infty}$ converges as $j \rightarrow \infty$, say, to $F^{*(k)}$. Write the nontrivial formal map $F^{*}\left(=\left(f^{*}, \phi^{*}, g^{*}\right)\right):=\sum_{k} F^{*(k)}$. By the way these maps were constructed, it is clear that $F^{*}$ satisfies the following normalization:

$$
\begin{aligned}
& f^{*}(z, w)=z+\text { terms with weighted degree higher than } 2, \\
& \phi^{*}(z, w)=\text { terms with degree higher than } 1,\left(\phi^{*}\right)^{(2,0)} \neq 0, \\
& g^{*}(z, w)=w+\text { terms with weighted degree higher than } 4 .
\end{aligned}
$$

Now $F^{*}$ is a formal map sending $M_{\ell}$ into $H_{\ell}^{n+2}$. According to a result of MeylanMir-Zaitsev [17], the formal map $F^{*}$ is indeed convergent. Hence, $F^{*}$ is a holomorphic map over $M_{\ell}$. Therefore, $F^{*}$ gives a CR immersion from $M_{\ell}$ into $H_{\ell}^{n+2}$. On the 
other hand, since any two CR transversal maps between a Levi-non-degenerate hypersurface and a hyperquadric of the same signature differ only by an automorphism of the hyperquadric ( see [8]), provided the codimension is less than $\frac{n}{2}$, we have a certain automorphism $T$ of $H_{\ell}^{n+2}$ such that near $p_{j} \approx 0$, and hence at all points in $M_{\ell}$ near the origin, it holds that

$$
F=T \circ F^{*}
$$

Since $T$ extends to an automorphism of the projective space $\mathbb{P}^{n+1}$ and $T(0)=0, F$ must be $\mathrm{CR}$ transversal at 0 . This is a contradiction. Our proof is complete.

Acknowledgement X. Huang was supported in part by NSF-1101481.

\section{References}

1. Baouendi, M.S., Ebenfelt, P., Rothschild, L.P.: Real Submanifolds in Complex Space and Their Mappings. Princeton Mathematics Series, vol. 47. Princeton University Press, Princeton (1999)

2. Baouendi, M.S., Ebenfelt, P., Rothschild, L.P.: Transversality of holomorphic mappings between real hypersurfaces in different dimensions. Commun. Anal. Geom. 15(3), 589-611 (2007)

3. Baouendi, M.S., Huang, X.: Super-rigidity for holomorphic mappings between hyperquadrics with positive signature. J. Differ. Geom. 69, 379-398 (2005)

4. Baouendi, M.S., Huang, X., Rothschild, L.P.: Nonvanishing of the differential of holomorphic mappings at boundary points. Math. Res. Lett. 2(6), 737-750 (1995)

5. Baouendi, M.S., Rothschild, L.P.: Geometric properties of mappings between hypersurfaces in complex space. J. Differ. Geom. 31(2), 473-499 (1990)

6. Chern, S.S., Moser, J.K.: Real hypersurfaces in complex manifolds. Acta Math. 133, 219-271 (1974)

7. D'Angelo, J.P.: Several Complex Variables and the Geometry of Real Hypersurfaces. Studies in Advanced Mathematics (1992)

8. Ebenfelt, P., Huang, X., Zaitsev, D.: The equivalence problem and rigidity for hypersurfaces embedded into hyperquadrics. Am. J. Math. 127(1), 169-191 (2005)

9. Ebenfelt, P., Huang, X., Zaitsev, D.: Rigidity of CR-immersions into spheres. Commun. Anal. Geom. 12(3), 631-670 (2004)

10. Ebenfelt, P., Rothschild, L.P.: Transversality of CR mappings. Am. J. Math. 128, 1313-1343 (2006)

11. Ebenfelt, P., Son, D.: Transversality of holomorphic mappings between real hypersurfaces in complex spaces of different dimensions, preprint (2011)

12. Fornaess, J.E.: Biholomorphic mappings between weakly pseudoconvex domains. Pac. J. Math. 74, 63-65 (1978)

13. Huang, X.: On a linearity problem for proper holomorphic maps between balls in complex spaces of different dimensions. J. Differ. Geom. 51, 13-33 (1999)

14. Huang, X.: Schwarz reflection principle in complex spaces of dimension two. Commun. Partial Differ. Equ. 21(11-12), 1781-1828 (1996)

15. Huang, X., Zhang, Y.: Monotonicity for the Chern-Moser-Weyl curvature tensor and CR embeddings. Sci. China Ser. A 52(12), 2617-2627 (2009)

16. Mir, N.: Convergence of formal embeddings between real-analytic hypersurfaces in codimension one. J. Differ. Geom. 62(1), 163-173 (2002)

17. Meylan, F., Mir, N., Zaitsev, D.: Approximation and convergence of formal CR-mappings. Int. Math. Res. Not. (4), 211-242 (2003)

18. Pinchuk, S.: Proper holomorphic maps of strictly pseudoconvex domains. Sib. Mat. Zh. 15, 909-917 (1974). Also see p. 959 (Russian)

19. Webster, S.: The rigidity of C-R hypersurfaces in a sphere. Indiana Univ. Math. J. 28(3), 405-416 (1979) 